\title{
Collective and Cooperative Group Behaviours: Biologically Inspired Experiments in Robotics
}

\author{
Alcherio Martinoli \\ Microcomputing Laboratory, Swiss Federal Institute of Technology \\ Lausanne, Switzerland \\ martinoli@di.epfl.ch \\ Francesco Mondada \\ Microcomputing Laboratory, Swiss Federal Institute of Technology \\ Lausanne, Switzerland \\ mondada@di.epfl.ch
}

\begin{abstract}
This paper describes the implementation of two biologically inspired collective behaviours on a group of Khepera miniature mobile robots. The first experiment is concerned with the gathering and clustering of randomly distributed small cylinders. In the second experiment the group of robots are expected to remove long sticks from holes, requiring a synchronous collaboration between two robots. The results are quantified, analysed and discussed, showing interesting (both positive and negative) aspects of this approach. Furthermore, we compare the results of both experiments with those reported by Deneubourg [1], [2] where similar behaviours are observed in ant colonies.
\end{abstract}

\section{Introduction}

In the last years we observe more and more collaborations between biologists and engineers [3]. For instance, common experiences, where biologically inspired control structures are implemented on real robots, allow biologists to understand how living organisms work, and engineers to develop new technologies that can deal with unsolved problems. This interaction between biologists and engineers is bringing new ideas to the traditional computational paradigms of robotics which typically involve several sequential and precise functional processes. First, sensing the environment, then detecting features, then constructing and modifying a world model, reasoning for the task and the world model in order to find some sequence of actions which might lead to success, then executing the action sequence one step at the time while updating the world model and replanning it if necessary at any stage. This is a very time consuming operation and requires a remarkable computational power and basic knowledge. 
the "subsumption" architecture [4]. The control architecture, inspired from some biological aspects, consists of a small number of simple modules, each capable to sense some limited aspect of the environment and to control part or all of the robot effector systems to achieve some limited tasks. Also in the field of neural networks, the biological inspiration plays an important role in the design of control architectures. At the Microcomputing Laboratory of the Swiss Institute of Technology many efforts has been undertaken to design and realize robots and control structures, based on biological models and to implement autonomous agents.

Let us now talk about the actual state of the research in a particular mutual domain of the biology and of the engineering fields: collective behaviour. Ant colonies are able to collect objects (such as food or dead ants) and place them in particular places. All ants of a given colony place the food at the same place and the carcass in another place. In this way they can collect and store food or carry dead ants to a "cemetery": if a large number of ant corpses or food particles are scattered outside a nest, they will pick them up, carry them for a while, and drop them. Within a short time we can observe that the corpses are being arranged into small clusters and, as time goes on, the number of clusters decreases and their size increases until eventually all the corpses will be in one or two large clusters. The emergence of these clusters has been studied with social insects by Deneubourg [1], who showed that a simple mechanism involving the modulation of the probability of dropping corpses as a function of the local density, was sufficient to generate the observed sequence of the clustering of corpses.

Gaussier and Zrehen [5] carried out an experiment with a group of Khepera robots implementing similar mechanisms with the same property: the probability of dropping corpses was a function of the local density. They mounted a hook behind the robot, which was therefore able, with an appropriate sequence of movements, to grasp and shift small cylindrical objects. Precise rules for the basic behaviours were defined: the perception of the objects and obstacles (Winner-Takes-All neuronal net) as well as dragging and placing objects were preprogrammed in such a way that the global probability of building a cluster was greater than that of destroying it. So, after a few minutes, the first clusters began to appear on the arena (see [5] for more details).

Beckers [6] made the same experiment with robots of approximatively $25 \mathrm{~cm}$ in diameter. The collective behaviour was analysed on the basis of the stigmergy principle, which signifies 'incitement to work by the products of the work'. It consists in essentially the production of a certain behaviour in agents as a consequence of the effects produced in the environment by previous actions. The experiment was carried out using 1 to 5 robots in an arena where many pucks of $4 \mathrm{~cm}$ in diameter were scattered. The robots were equipped with a frontal surface to push the pucks. A microswitch was installed behind the surface to control the maximal number of pucks which can be pushed at the same time. The robot was able to shift two pucks, but when three pucks were detected by the microswitch, the robot stopped pushing and changed direction. Their results indicated that the optimal density of robots on the arena surface, in order to accomplish the given collective task in a minimal time lapse (relative to the number of robots), was three. According to the authors, the reasons for the presence of this optimum were attributed to the geometry of the clusters and to the constructive and destructive interferences among the agents. 
behaviour of a colony of ants. They rammed some sticks close to the nest and they observed what occurred. After a few minutes, the ants tried to grasp the sticks in order to accumulate building material for their nest, but a single ant was not able to accomplish this task. A few minutes later, the colony solved the problem with a collaborative behaviour among two or more ants.

We can classify the above mentioned experiments into two categories, considering the different tasks involved:

- collective noncooperative behaviour, which does not necessarily need cooperation among the individuals to be solved, i.e., a greater number of agents can only accelerate the work to be accomplished;

- collective cooperative behaviour, which absolutely needs the collaboration of two or more individuals in order to be carried out, because of some physical constraints of a single agent.

This paper aims to describe two experiments on real robots, which can be classed into the two above mentioned categories. The discussion will also focus on the radical difference between the two tasks.

\section{Materials and Methods}

Khepera is a miniature mobile robot developed to perform "desktop" experiments [7]. Its main characteristic is a diameter of $55 \mathrm{~mm}$. It also has other very interesting possibilities, such as an important processing power (32 bits processor at $16 \mathrm{MHz}$ ), autonomy, precise odometry, light and proximity sensors, grasping possibilities, and many other additional features that can be added on a extension bus. In its basic configuration Khepera is equipped with 8 infrared (IR) sensors, 6 on the front and 2 behind its cylindrical structure. On the front these sensors are distributed with a gap of about $14 \mathrm{~mm}$. The wheels are controlled by two DC motors with an incremental encoder (12 pulses per mm of advancement of the robot), and can move in both directions. The simple geometrical shape and the motor layout allow Khepera to negotiate any kind of obstacle or corner. Each robot is extended with a gripper module, which can grasp and carry objects with a maximum diameter of $50 \mathrm{~mm}$ (see fig. 2a). A robot of this size is not only optimal for the test of basic features in an individual behaviour, but is also a very interesting platform with which to perform experiments in collective robotics: 20 robots can easily work on $2 \mathrm{~m}^{2}$, which approximately represents an area of $10 \times 20 \mathrm{~m}$ for a bigger robot (being for instance $50 \mathrm{~cm}$ in diameter).

Our two experiments on collective behaviour use 1 to 5 Kheperas. In both cases, the number of robots involved has been gradually increased and the performance measured and compared. The objects manipulated by the robot in the first experiment have a cylindrical form, with a diameter of $16 \mathrm{~mm}$ and a height of $25 \mathrm{~mm}$. In the second experiment, we use longer cylinders $(150 \mathrm{~mm})$ with the same diameter as before; they stretch out $50 \mathrm{~mm}$ from the ground (see fig. 6b). Both experiments are carried out in a square arena of $80 \times 80 \mathrm{~cm}$. The initial scattering of the objects is arbitrarily predefined. Our experiments are repeated and quantified 3 times and each experiment takes approximately 30 minutes.

It is worth emphasising that in both experiments the robots operate completely autonomously and independently; all sensors, motors and controls are on-board, 


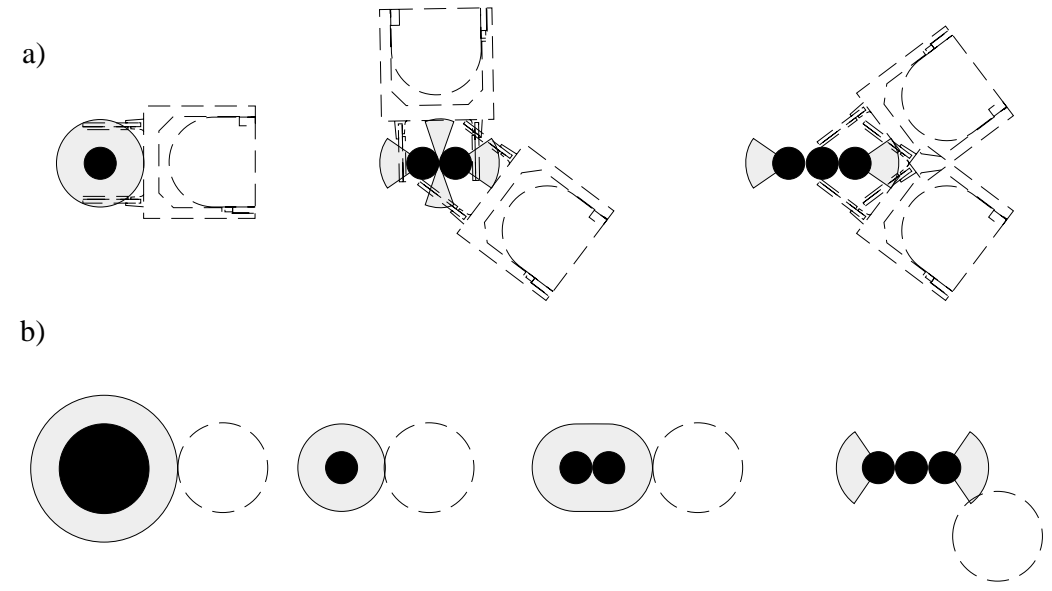

Figure 4. Geometrical representation of access areas for Khepera in order to destroy (a) a cluster of one object, a clusters of two objects and a clusters of three objects. b) Access areas for Khepera in order to build a group of one object from nothing (wrong detection of another robot), to build a group of two objects from one object, a group of three objects from a group of two and a group of four objects from a group of three.

experiment, we notice that a major density of robots on the arena causes only an increasing rate of destructive interferences (e.g., avoidance of the other robots, false perception of the object to grasp), as demonstrated in the first and more reliable part of the experiment. Therefore, the results of this experiment contribute to the proof that a collective but noncooperative task can not always be achieved with better performances (in this case the judgement criterion was the relative speed) increasing the density of robots.

Let us analyse in more detail the process rules of the first experiment. Figure 4 presents a geometrical explanation of the building-destroying mechanism. We draw the access area in order to perform one action or the other. We consider the cluster and the robot gripper geometry. The cluster building area is always greater than the cluster destroying area. The size of these areas is directly proportional to a sort of mean global probability; hence, we can assert that the building process is more probable than the destroying one and the result is, as illustrated by figure $3 \mathrm{a}$, a final dynamical equilibrium.

In figure 5, the normalised results of figure 4 are presented. The probability of building a two objects cluster from two single object clusters (i.e., destroying them) is very high. On the other hand the probability to build greater clusters is inversely proportional to their size. In effect, it is a question of conditional probability: an 8 objects cluster can only be built if a 7 objects cluster previously exists.

\subsection{Second Experiment}

The setup of the second experiment is depicted in figure 6. The event showed in figure $6 \mathrm{c}$ has occurred almost every 20 minutes. How we mentioned above, this experiment implements a collective cooperative task, this means that the stick can not be removed by a single Khepera. Because of the very low rate of collaboration, it is difficult to formulate any consideration concerning the optimal density of robots on the arena. Furthermore, there are more destructive interferences during this 
the contrary, the collective aspect introduces many destructive interactions, such as collisions between robots, errors of detection due to moving obstacles, conflicting situations, etc. Probably the single robot performance, which represents the top limit in this type of task and with this kind of performance measurement, can only be improved by groups of robots if some kind of differentiation of the individual behaviour is introduced.

The results of the second experiment show that a good interaction between the robots and the environment, also with a very simple behaviour, can give rise to a collective task where a real cooperation is present.

These two experiments show interesting characteristics of collective performances where the individual behaviour is very simple and the same on every robot of the group. The interactions between many robots and between the robots and the environment play a crucial role in the performances of the group. A very simple modification of these relationships, as illustrated in the two experiments, can radically modify the performances and the behaviour of the group. Both types of working organisations (cooperative and noncooperative) can be very useful in robotics to extend the capacity of a single robot. To exploit this group structure in new design methodologies and apply it to real useful applications, we still need to better understand the basic mechanisms of collective work.

\section{Acknowledgements}

We would like to thank Edo Franzi and André Guignard for the important work in the design of Khepera, and Paolo Ienne for the review of this paper. Alcherio Martinoli and Francesco Mondada have been partially supported by the Swiss National Research Foundation (project FN and PNR23).

\section{References}

[1] J. C. Deneubourg, S. Goss, N. Franks, A. Sendova, A. Franks, C. Detrin, and L. Chatier. The dynamics of collective sorting: Robot-like ant and ant-like robot. In J. A. Mayer and S. W. Wilson, editors, Simulation of Adaptive Behavior: From Animals to Animats, pages 356-365. MIT Press, 1991.

[2] J.C. Deneubourg, 1994. Personal Communication.

[3] J. C. Deneubourg, P. S. Clip, and S. S. Camazine. Ants, buses and robots selforganization of transportation systems. In P. Gaussier and J-D. Nicoud, editors, Proceedings of the conference From Perception to Action. IEEE Press, Los Alamitos, CA, 1994.

[4] R. A. Brooks. A robust layered control system for a mobile robot. IEEE Robotics and Automation, RA-2:14-23, March 1986.

[5] P. Gaussier and S. Zrehen. A constructivist approach for autonomous agents. In Thalmann D. \& N., editor, Artificial Life in Virtual Reality, pages 97-113. John Wiley and Sons, London, 1994.

[6] R. Beckers, O.E. Holland, and J.L. Deneubourg. From local actions to global tasks: Stigmergy and collective robotics. In R. Brooks and P. Maes, editors, Proceedings of the Fourth Workshop on Artificial Life, Boston, MA, 1994. MIT Press.

[7] F. Mondada, E. Franzi, and P. Ienne. Mobile robot miniaturization: A tool for investigation in control algorithms. In Proceedings of the Third International Symposium on Experimental Robotics, Kyoto, Japan, 1993. 